\title{
Comparison of Phase-Sensitive Inversion Recovery and Conventional Magnetic Resonance Imaging for Detection of Cortical Plaques in MS
}

\section{Patients}

\author{
Hassan Hashemi ${ }^{1}$, Maryam Mohammadzadeh (iD ${ }^{2}$, Mohammad Hossein Dianat ${ }^{1}$, Amir Reza Azimi ${ }^{3}$, \\ Hamed Naghibi ${ }^{1}$, Madjid Shakiba ${ }^{1}$ and Kavous Firouznia ${ }^{1, *}$ \\ ${ }^{1}$ Advanced Diagnostic and Interventional Radiology Research Center (ADIR), Tehran University of Medical Sciences, Tehran, Iran \\ ${ }^{2}$ Department of Radiology, Division of Neuroradiology, Amiralam Hospital, Tehran University of Medical Sciences, Tehran, Iran \\ ${ }^{3}$ MS Research Center, Neuroscience Institute, Tehran University of Medical Sciences, Tehran, Iran \\ "Corresponding author: Advanced Diagnostic and Interventional Radiology Research Center (ADIR), Tehran University of Medical Sciences, Tehran, Iran. Email: \\ k_firouznia@yahoo.com \\ Received 2020 December 13; Revised 2021 May 07; Accepted 2021 June 14.
}

\begin{abstract}
Background: Precise detection and classification of intracortical (IC) lesions in multiple sclerosis (MS) patients are very important for understanding their role in disease progression and determining their effects on the clinical presentations of the disease.

objectives: This study aimed to evaluate the efficacy of phase-sensitive inversion recovery (PSIR) in delineation of cortical lesions in MS patients.

Patients and Methods: This cross-sectional, single-center study was performed among 38 patients with the mean age of 31 years, who were recruited from December 2018 to August 2020. All MS patients underwent magnetic resonance imaging (MRI), using a 1.5-Tesla scanner. Two expert neuroradiologists interpreted the fluid-attenuated inversion recovery (FLAIR), T2-weighted turbo spin echo (T2W-TSE), and PSIR images. The lesions were classified as purely IC, mixed gray/white matter (WM) [leukocortical (LC)], and juxtacortical (JC). The number of lesions in each region was compared between the FLAIR, T2W-TSE, and PSIR sequences.

Results: The number of cortical lesions (IC and LC) was significantly higher in PSIR compared to T2W-TSE and Fluid attenuated inversion recovery (FLAIR) $(\mathrm{P}<0.001)$, while the number of JC lesions was lower; in other words, the mean number of plaques was higher in T2W-TSE and FLAIR as compared to PSIR.

Conclusion: The PSIR sequence significantly improved the delineation of cortical lesions and could be useful in monitoring cortical injuries and disease progression in MS patients.
\end{abstract}

Keywords: Magnetic Resonance Imaging, Multiple Sclerosis, PSIR, Cortical Lesion

\section{Background}

Magnetic resonance imaging (MRI) is perhaps the single most important imaging modality for the diagnosis of multiple sclerosis (MS). MRI, which is widely used to monitor the disease activity, plays an important role in establishing the clinical diagnosis of MS. The gray matter (GM) lesions are common findings in the neuropathological studies of MS $(1,2)$. However, they are not easily delineated in conventional MR sequences because of their small size and lack of contrast between cortical lesions (CLs) and the surrounding GM.

Although routine MRI using T2-weighted (T2W) and fluid-attenuated inversion recovery (FLAIR) sequences may demonstrate CLs, these sequences are considerably less sensitive for the detection of CLs as compared to white matter $(\mathrm{WM})$ plaques $(3,4)$. Overall, the limited detection of
CLs influences our understanding of the impact of cortical plaques on the clinical presentations of MS patients, such as cognitive impairment, sensory loss (i.e., paresthesia), ataxia, tremors, and affective disorders (5-7).

Multiple MRI sequences have been developed at various field strengths to improve the visualization of CLs. FLAIR sequence is commonly used to assess WM lesions, especially those located in the brain. Although FLAIR is a T2W-based sequence, because it has the ability to suppress the cerebrospinal fluid (CSF) signals, it is preferred to T2Wturbo spin echo (TSE) for delineation of periventricular MS plaques; however, this modality fails to accurately visualize CLs (4). Besides, double inversion recovery (DIR) is the most widely applied sequence for manifestation of cortical plaques (2). In 2001, DIR was the first reported sequence to identify CLs along with MRI. This sequence suppresses both 
WM and CSF signals, leading to the improved conspicuity of demyelinating plaques in both WM and GM regions of the brain compared to T2W-TSE and FLAIR images (8).

Many studies have suggested a relationship between CLs delineated on DIR and the physical and cognitive impairments of MS patients and found cortical plaques to be clinically relevant $(9,10)$. However, the DIR sequence is generally prone to image artifacts, such as flow, which may affect the visualization of subtle intracortical (IC) lesions. It also shows regional variations in the GM signal intensity, possibly leading to the false-positive detection of lesions. Besides, DIR does not visualize the lesion borders precisely, and its low signal-to-noise ratio (SNR) further obscures small lesions (10).

Recently, phase-sensitive inversion recovery (PSIR), a T1weighted sequence with a higher SNR and GM/WM contrast, has been shown to improve the detection and classification of CLs using DIR in MS patients (1-3). This technique has been used for the diagnosis of cervical cord MS plaques (11), although it has been recently applied for MS patients, as well. Overall, PSIR allows for a better classification of lesions into purely IC and subcortical types (WM lesions) (2).

\section{Objectives}

The present study aimed to compare the efficacy of T2W-TSE, FLAIR, and PSIR MR sequences in identifying CLs in MS patients. The number of lesions in each sequence was determined to show which sequence identified more lesions in the patients.

\section{Patients and Methods}

\subsection{Study Population}

This cross-sectional study was conducted on 38 patients with a confirmed diagnosis of relapsing-remitting MS (RR-MS), who underwent brain MRI from December 2018 to August 2020. The inclusion criteria were age above 18 years and a definite diagnosis of RR-MS, as defined by the McDonald criteria (2017) (12). Patients with MRI contraindications were not included. On the other hand, the exclusion criteria were as follows: (1) other major medical, neurological, or neuropsychiatric disorders in the patient's medical history; and (2) incomplete MRI exam, defined as the absence of one or several sequences. The study protocol was approved by the local ethics review board, and all subjects provided a written informed consent form before the study. The patients' characteristics are summarized in Table 1.

\subsection{Imaging Protocol}

The patients were scanned on a 1.5-T MRI scanner (MAGNETOM Symphony, Siemens, Erlangen, Germany) with a six-channel head coil. The PSIR images were obtained for each patient in every session as part of our routine MRI sequences, which also included conventional T2W-TSE and FLAIR. The relevant sequence parameters are presented in Table 2 .

\subsection{Image Analysis}

Two independent neuroradiologists separately assessed all MR images. The number of plaques was determined in each sequence and compared blindly among sequences. The plaques were defined as hyperintense in T2W and FLAIR and as hypointense in PSIR. The number of plaques was also determined in terms of IC, leukocortical (LC), and juxtacortical (JC) regions. The IC lesions were confined to the GM, while lesions that involved both the cortex and the adjacent WM were identified as LC lesions. Finally, the JC lesions were WM lesions involving the subcortical U-fibers.

\subsection{Statistical Analysis}

The plaque count in different sequences is expressed as mean \pm SD. Although this parameter does not have a decimal, we expressed it with a decimal for a better comparison between the groups [25 - 75 interquartile range (IQR)]. The plaque number was compared between the three different protocols by Friedman test. Also, a pairwise comparison between the groups was carried out using Wilcoxon signed-rank test, based on Bonferroni correction. Moreover, for nominal variables, the three groups were compared by Cochran's Q test. For pairwise comparisons, McNemar's test was performed based on the Bonferroni correction. A P-value of 0.05 was considered to be significant for comparisons using Friedman test, Cochran's Q test, and other tests. For corrected P-values in Bonferroni correction, since we aimed to perform three post-hoc comparisons (T2 vs. FLAIR, T2 vs. PSIR, and FLAIR vs. PSIR), the significance level was set at 0.017 (0.05/3).

Because two neuroradiologists assessed all of the patients, and the results were reported separately, the interrater reliability was not a major issue. Also, for intra-rater reliability, we asked the two neuroradiologists to blindly assess 15 patients after two weeks. Next, we calculated the intra-rater reliability by measuring the intraclass correlation coefficient (ICC) and Kappa's coefficient separately. All indices showed acceptable values (all above 0.75). SPSS version 18 (released in 2009, PASW Statistics for Windows, SPSS Inc., Chicago, IL, USA) was used for data analysis. P-values less than 0.05 were considered statistically significant. 


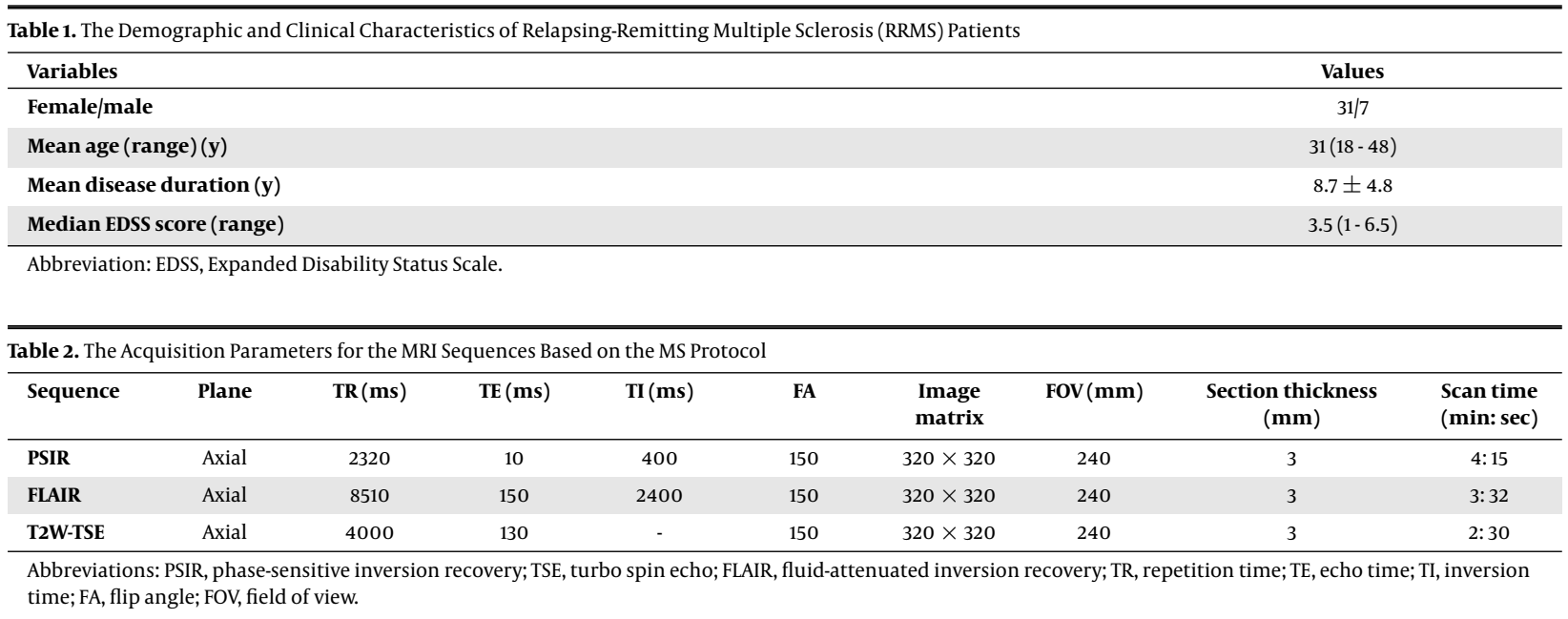

\section{Results}

Thirty-eight RR-MS patients were included in this study. The mean age of the patients was 31 years (range: 18 - 48 years), and the median Expanded Disability Status Scale (EDSS) score was 3.5 (range: 1 - 6.5). The mean plaque number was compared between the three MRI sequences for each radiologist. Besides, the sum of lesions and the mean of results reported by the two neuroradiologists were compared between the sequences (Table 3 ).

The mean number of plaques in the IC region based on PSIR was higher than that found on FLAIR and T2WTSE for both neuroradiologists $(\mathrm{P}<0.001)$; a similar pattern was seen in the LC region $(\mathrm{P}<0.001)$. However, the mean number of plaques in the JC region on T2W-TSE and FLAIR was higher than PSIR for both neuroradiologists ( $P$ $<0.001)$. Considering the mean count, the two neuroradiologists reported similar findings. Also, considering the sum of plaques in the three regions, the highest mean was reported for PSIR, followed by T2W-TSE and FLAIR, respectively; this pattern was similar for both neuroradiologists.

Besides the mean number of plaques, we performed pairwise comparisons of sequences in each anatomic site to determine the number of patients with a higher, similar, or lower number of plaques. For example, based on the data reported by the first radiologist, T2W-TSE and FLAIR were compared in the LC region. We found that three patients had more lesions on FLAIR than T2W-TSE; six patients had more lesions on T2W-TSE than FLAIR; and seven patients had an equal number of lesions in both sequences (22 cases without plaques in both T2W-TSE and FLAIR). A similar analysis was performed for the pairwise comparison of all anatomic sites for both neuroradiologists; the results are presented in Table 4 . In each pairwise comparison regarding the number and percentage of plaques, a significant P-value shows which sequence can show more plaques using a patient-based approach (Table 4).

In both IC and LC regions, most patients showed more plaques in PSIR compared to FLAIR and T2W-TSE, while in the JC region, most patients had more plaques in $\mathrm{T} 2 \mathrm{~W}$ TSE and FLAIR compared to PSIR (Figure 1); similar findings were reported by both neuroradiologists. Using another approach, we considered the presence or absence of plaques and performed comparisons, as presented in Tables 1 and 2 . The patterns of statistical significance are similar to those presented in Table 5 and Figure 2.

\section{Discussion}

The present study aimed to assess the application of PSIR sequence compared to conventional sequences, including T2W-TSE and FLAIR, for the detection of CLs in MS patients. Based on the results, PSIR was superior to conventional sequences in the detection of cortical MS lesions in the IC and LC regions. Previous studies have shown that almost one-third of MS patients have at least one plaque or a lesion in the cortex, which may not be detected in conventional sequences (13).

Occasionally, IC plaques can be seen in the early stages of MS, although they usually occur in the advanced stages of the disease. They are found in patients with severe brain damage and can increase clinical disability (14). As CLs can adversely affect neurological and cognitive activities, their diagnosis plays an important role in the patients' prognosis (15). It seems that a better delineation of the GM/WM border on PSIR sequences allows for a more precise classification of lesions into purely IC, LC, and JC.

Considering the inherent features of the PSIR sequence, image production is dependent on phase of the protons In this image, the tissue, nulled by the inversion time, is displayed as intermediate gray, while all other tissues have a lower or higher signal intensity, depending 


\begin{tabular}{|c|c|c|c|c|c|c|c|}
\hline \multirow{2}{*}{ Variables } & \multirow{2}{*}{$\begin{array}{c}\text { Median }[25-75 \text { th } \\
\text { percentile] }\end{array}$} & \multirow{2}{*}{ Mean \pm SD } & \multirow{2}{*}{ Range } & \multicolumn{4}{|c|}{ P-value } \\
\hline & & & & $\begin{array}{l}\text { Three } \\
\text { groups }\end{array}$ & $\begin{array}{l}\text { T2W-TSE vs. } \\
\text { FLAIR }\end{array}$ & $\begin{array}{l}\text { T2W-TSE vs. } \\
\text { PSIR }\end{array}$ & $\begin{array}{l}\text { FLAIR vs. } \\
\text { PSIR }\end{array}$ \\
\hline \multicolumn{8}{|l|}{ First radiologist } \\
\hline IC & & & & $<0.001$ & 0.32 & 0.002 & 0.002 \\
\hline T2W-TSE & $0[0-0]$ & 0 & 0 & & & & \\
\hline FLAIR & $0[0-0]$ & $0.03 \pm 0.16$ & $0-1$ & & & & \\
\hline PSIR & $0[0-1]$ & $0.63 \pm 1.57$ & $0-9$ & & & & \\
\hline LC & & & & $<0.001$ & 0.71 & $<0.001$ & $<0.001$ \\
\hline T2W-TSE & $0[0-1]$ & $0.39 \pm 0.59$ & $0-2$ & & & & \\
\hline FLAIR & $0[0-1]$ & $0.34 \pm 0.58$ & $0-2$ & & & & \\
\hline PSIR & $1[0-2.25]$ & $1.45 \pm 1.84$ & $0-7$ & & & & \\
\hline JC & & & & $<0.001$ & 0.004 & $<0.001$ & 0.047 \\
\hline T2W-TSE & $2[0-3]$ & $2.29 \pm 2.63$ & $0-10$ & & & & \\
\hline FLAIR & $1[0-3]$ & $1.79 \pm 2.24$ & $0-9$ & & & & \\
\hline PSIR & $1[0-2]$ & $1.32 \pm 1.54$ & $0-7$ & & & & \\
\hline \multicolumn{8}{|l|}{ Second radiologist } \\
\hline IC & & & & $<0.001$ & 0.18 & 0.001 & 0.001 \\
\hline T2W-TSE & $0[0-0]$ & $0.03 \pm 0.16$ & $0-1$ & & & & \\
\hline FLAIR & $0[0-0]$ & $0.11 \pm 0.39$ & $0-2$ & & & & \\
\hline PSIR & $0[0-1]$ & $0.61 \pm 1.52$ & $0-9$ & & & & \\
\hline LC & & & & $<0.001$ & 0.13 & $<0.001$ & $<0.001$ \\
\hline T2W-TSE & $0[0-1]$ & $0.50 \pm 0.73$ & $0-3$ & & & & \\
\hline FLAIR & $0[0-1]$ & $0.32 \pm 0.57$ & $0-2$ & & & & \\
\hline PSIR & $1[0-2.25]$ & $1.92 \pm 2.34$ & $0-9$ & & & & \\
\hline JC & & & & $<0.001$ & 0.21 & $<0.001$ & 0.004 \\
\hline T2W-TSE & $2[0-3.25]$ & $2.47 \pm 2.97$ & $0-12$ & & & & \\
\hline FLAIR & $1[0-2.25]$ & $2.18 \pm 2.97$ & $0-13$ & & & & \\
\hline PSIR & $1[0-2]$ & $1.26 \pm 1.72$ & $0-8$ & & & & \\
\hline $\begin{array}{l}\text { Sum of the three regions reported by the first } \\
\text { radiologist }\end{array}$ & & & & $<0.001$ & 0.004 & $<0.023$ & $<0.001$ \\
\hline T2W-TSE & $2[1-3.25]$ & $2.68 \pm 3.05$ & $0-12$ & & & & \\
\hline FLAIR & $1.5[1-3]$ & $2.16 \pm 2.33$ & $0-10$ & & & & \\
\hline PSIR & $2[1-5]$ & $3.39 \pm 4.17$ & $0-21$ & & & & \\
\hline $\begin{array}{l}\text { Sum of the three regions reported by the second } \\
\text { radiologist }\end{array}$ & & & & $<0.001$ & 0.008 & 0.001 & $<0.001$ \\
\hline T2W-TSE & $2[1-4]$ & $3.00 \pm 3.46$ & $0-14$ & & & & \\
\hline FLAIR & $2[0-3.25]$ & $2.61 \pm 3.14$ & $0-13$ & & & & \\
\hline PSIR & $2.5[1-5]$ & $3.79 \pm 4.45$ & $0-19$ & & & & \\
\hline \multicolumn{8}{|l|}{ Mean of results reported by the two radiologists } \\
\hline IC & & & & $<0.001$ & 0.18 & $<0.001$ & $<0.001$ \\
\hline T2W-TSE & $0[0-0]$ & $0.01 \pm 0.08$ & $0-0.5$ & & & & \\
\hline FLAIR & $0[0-0]$ & $0.07 \pm 0.26$ & $0-1.5$ & & & & \\
\hline PSIR & $0[0-1]$ & $0.62 \pm 1.23$ & $0-6$ & & & & \\
\hline \multicolumn{8}{|l|}{ Mean of results reported by the two radiologists } \\
\hline LC & & & & $<0.001$ & 0.20 & $<0.001$ & $<0.001$ \\
\hline T2W-TSE & $0.25[0-1]$ & $0.45 \pm 0.58$ & $0-2.5$ & & & & \\
\hline FLAIR & $0[0-0.5]$ & $0.33 \pm 0.51$ & $0-2$ & & & & \\
\hline PSIR & $1[0-2.625]$ & $1.68 \pm 2.06$ & $0-8$ & & & & \\
\hline \multicolumn{8}{|l|}{ Mean of results reported by the two radiologists } \\
\hline JC & & & & $<0.001$ & 0.01 & $<0.001$ & 0.006 \\
\hline T2W-TSE & $1.5[0-3]$ & $2.38 \pm 2.77$ & $0-11$ & & & & \\
\hline FLAIR & $1.5[0.375-2.625]$ & $1.99 \pm 2.58$ & $0-11$ & & & & \\
\hline PSIR & $1[0-2]$ & $1.29 \pm 1.57$ & $0-6.5$ & & & & \\
\hline
\end{tabular}

Abbreviations: PSIR, phase-sensitive inversion recovery; FLAIR, fluid-attenuated inversion recovery; TSE, turbo spin echo; IC, intracortical lesion; LC, leukocortical lesion; JC, juxtacortical lesion. 

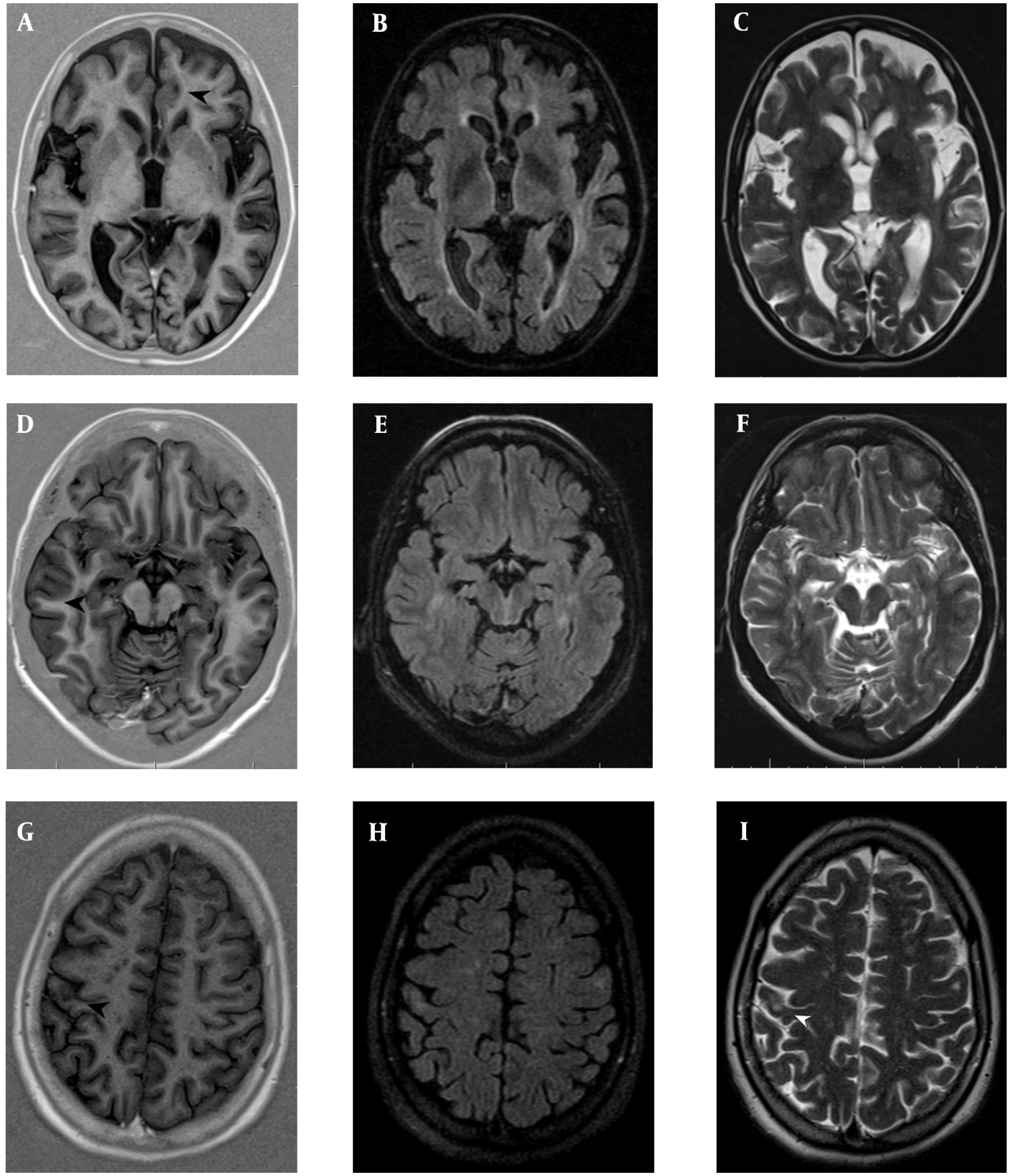

Figure 1. Several patients with relapsing-remitting multiple sclerosis (RRMS) showing focal lesions in the cortical grey matter A B \& $C$. Intracortical (IC); and D, E \& F, Leukocortical (LC) plaques were counted on PSIR (A,D) (black head arrows), but were not identified on FLAIR (B,E) and T2TSE (C,F). G, H \& I, The juxtacortical (JC) lesions were observed better on, T2TSE (I) (white head-arrow) and FLAIR (H) than PSIR (G) (black head-arrow). PSIR, phase-sensitive inversion recovery; FLAIR, fluid-attenuated inversion recovery; TSE, turbo spin echo. 




Abbreviations: PSIR, phase-sensitive inversion recovery; FLAIR, fluid-attenuated inversion recovery; TSE, turbo spin echo; IC, intracortical lesion; LC, leukocortical lesion; JC, juxtacortical lesion.

on their $\mathrm{T} 1$ relaxation time relative to $\mathrm{T} 1$ relaxation time of the nulled tissue; therefore, its grayscale criteria have a larger amplitude (16). The phase-sensitive reconstruction improves the GM/WM contrast and differentiation. Besides, it makes the CSF appear hypointense, as it produces a large negative magnetization field (16). According to a pervious study, CLs can appear hyperintense on T2W-TSE and
FLAIR images. Also, blood and CSF flow artifacts appear hyperintense in such images, and the false positive probability increases; conversely, these lesions appear hypointense in PSIR sequences (4).

Our study showed that the total number of MS IC and LC plaques was significantly higher in PSIR compared to T2W-TSE and FLAIR sequences. This finding is similar to the 


\begin{tabular}{|c|c|c|c|c|c|c|c|c|c|}
\hline Variables/T2W-TSE vs. FLAIR & No. (\%) & Overall P & $\mathbf{P}$ & T2W-TSE vs. PSIR & No. (\%) & $\mathbf{P}$ & FLAIR vs. PSIR & No. (\%) & $\mathbf{P}$ \\
\hline \multicolumn{10}{|l|}{ First radiologist } \\
\hline IC & & $<0.001$ & 0.32 & & & $<0.001$ & & & 0.001 \\
\hline Both yes & 0 & & & Both yes & 0 & & Both yes & $1(2.6)$ & \\
\hline FLAIR yes, T2W-TSE no & $1(2.6)$ & & & PSIR yes, T2W-TSE no & $12(31.6)$ & & PSIR yes, FLAIR no & $11(28.9)$ & \\
\hline FLAIR no, T2W-TSE yes & 0 & & & PSIR no, T2W-TSE yes & 0 & & PSIR no, FLAIR yes & 0 & \\
\hline Both no & $37(97.4)$ & & & Both no & $26(68.4)$ & & Both no & $26(68.4)$ & \\
\hline LC & & $<0.001$ & 0.73 & & & 0.002 & & & 0.002 \\
\hline Both yes & $8(21.1)$ & & & Both yes & $13(34.2)$ & & Both yes & $10(26.3)$ & \\
\hline FLAIR yes, T2W-TSE no & $3(7.9)$ & & & PSIR yes, T2W-TSE no & $10(26.3)$ & & PSIR yes, FLAIR no & $13(34.2)$ & \\
\hline FLAIR no, T2W-TSE yes & $5(13.2)$ & & & PSIR no, T2W-TSE yes & 0 & & PSIR no, FLAIR yes & $1(2.6)$ & \\
\hline Both no & $22(57.9)$ & & & Both no & $15(39.5)$ & & Both no & $14(36.8)$ & \\
\hline JC & & 0.37 & 0.9 & & & 0.5 & & & 0.63 \\
\hline Both yes & $26(68.4)$ & & & Both yes & $25(65.8)$ & & Both yes & $24(63.2)$ & \\
\hline FLAIR yes, T2W-TSE no & $1(2.6)$ & & & PSIR yes, T2W-TSE no & 0 & & PSIR yes, FLAIR no & $1(2.6)$ & \\
\hline FLAIR no, T2W-TSE yes & $1(2.6)$ & & & PSIR no, T2W-TSE yes & $2(5.3)$ & & PSIR no, FLAIR yes & $3(7.9)$ & \\
\hline Both no & $10(26.3)$ & & & Both no & $11(28.9)$ & & Both no & $10(26.3)$ & \\
\hline \multicolumn{10}{|l|}{ Second radiologist } \\
\hline IC & & $<0.001$ & 0.5 & & & $<0.001$ & & & 0.002 \\
\hline Both yes & $1(2.6)$ & & & Both yes & $1(2.6)$ & & Both yes & $3(7.9)$ & \\
\hline FLAIR yes, T2W-TSE no & $2(5.3)$ & & & PSIR yes, T2W-TSE no & $12(31.6)$ & & PSIR yes, FLAIR no & $10(26.3)$ & \\
\hline FLAIR no, T2W-TSE yes & 0 & & & PSIR no, T2W-TSE yes & 0 & & PSIR no, FLAIR yes & 0 & \\
\hline Both no & $35(92.1)$ & & & Both no & $25(65.8)$ & & Both no & $25(65.8)$ & \\
\hline LC & & $<0.001$ & 0.23 & & & 0.003 & & & $<0.001$ \\
\hline Both yes & $7(18.4)$ & & & Both yes & $14(36.8)$ & & Both yes & $8(21.1)$ & \\
\hline FLAIR yes, T2W-TSE no & $3(7.9)$ & & & PSIR yes, T2W-TSE no & $12(31.6)$ & & PSIR yes, FLAIR no & $18(47.4)$ & \\
\hline FLAIR no, T2W-TSE yes & $8(21.1)$ & & & PSIR no, T2W-TSE yes & $1(2.6)$ & & PSIR no, FLAIR yes & $2(5.3)$ & \\
\hline Both no & $20(52.6)$ & & & Both no & $11(28.9)$ & & Both no & $10(26.3)$ & \\
\hline JC & & 0.005 & 0.9 & & & 0.016 & & & 0.03 \\
\hline Both yes & $26(68.4)$ & & & Both yes & $21(55.3)$ & & Both yes & $21(55.3)$ & \\
\hline FLAIR yes, T2W-TSE no & $1(2.6)$ & & & PSIR yes, T2W-TSE no & 0 & & PSIR yes, FLAIR no & 0 & \\
\hline FLAIR no, T2W-TSE yes & $2(5.3)$ & & & PSIR no, T2W-TSE yes & $7(18.4)$ & & PSIR no, FLAIR yes & $6(15.8)$ & \\
\hline Both no & $9(23.7)$ & & & Both no & $10(26.3)$ & & Both no & $11(28.9)$ & \\
\hline
\end{tabular}

Abbreviations: PSIR, phase-sensitive inversion recovery; FLAIR, fluid-attenuated inversion recovery; TSE, turbo spin echo; IC, intracortical lesion; LC, leukocortical lesion; JC, juxtacortical lesion.

a"yes" in table means "show/showes plaque" and "no" in table means "don't show/doesn't show plaque".

results of a study conducted by Nelson et al. (3), which compared the number of lesions detected by PSIR, FLAIR, and DIR sequences. Moreover, in a study by Sethi et al. (2), the implementation of PSIR sequence significantly increased the number of additional plaques in the IC region. In another study by Favaretto et al. (17), the PSIR sequence could identify lesions four times more than DIR, especially IC and
LC lesions. Another study by Harel et al. (1) verified that PSIR improves the detection and classification of CLs as compared to DIR; this study showed that CLs affected the physical and cognitive disabilities of patients with MS.

In the present study, PSIR showed significantly more MS lesions in the IC and LC regions compared to T2W-TSE and FLAIR sequences. The mean number of lesions in these 

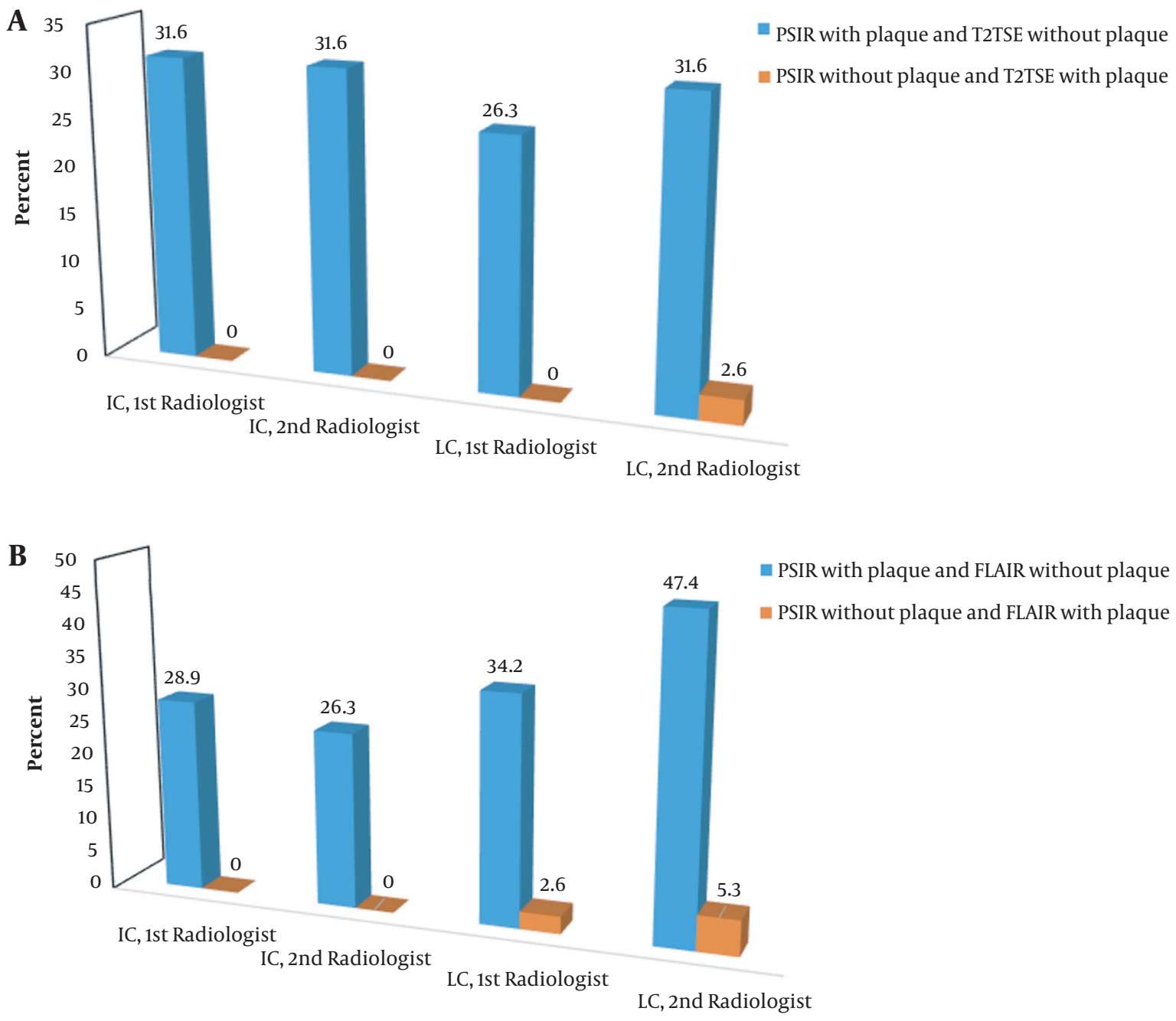

Figure 2. A, Comparison of PSIR with T2W-TSE: the distribution of patients in subgroups based on the presence of plaque in one sequence and its absence from another sequence (based on the radiologist's observation and location); B, Comparison of PSIR with FLAIR: the distribution of patients in subgroups based on the presence of plaque in one sequence and its absence from another sequence (based on the radiologist's observation and location). PSIR, phase-sensitive inversion recovery; FLAIR, fluid-attenuated inversion recovery; TSE, turbo spin echo; IC, intracortical lesion; LC, leukocortical lesion.

two regions was greater in PSIR as compared to FLAIR and T2W-TSE, and the difference was statistically significant ( $P$ $<0.001$ ). Besides, a higher number of lesions was found in the JC region by T2W-TSE compared to PSIR and FLAIR ( $<$ 0.001). This finding is consistent with the results reported by Wattjes et al. (18), which also indicated more lesions in the JC region using FLAIR images; it should be noted that many lesions in the JC region are WM lesions rather than CLs, which are easily detected by FLAIR and T2W-TSE.

According to previous studies, the use of both DIR and PSIR sequences can improve the detection of MS CLs (4). In this regard, Sethi et al. (19) showed that the higher CNR, provided by the PSIR sequence, led to the improved detec- tion and anatomic classification of GM lesions compared to other sequences, such as DIR. The cortical GM/WM junction is typically missed on conventional images used to distinguish between IC lesions and those extending into the WM (considered as LC lesions). On the other hand, the PSIR sequence creates a high contrast between the lesion and the adjunct MG, leading to the delineation of the lesion size and boundaries.

The diagnosis of MS according to the McDonald criteria is based on the lesion dissemination in time and space (in anatomic sites, including the JC, periventricular, infratentorial, IC, and spinal cord regions). In other words, at least two lesions in two anatomic sites fulfill the criteria 
for dissemination in space (12). Therefore, even a patient who shows, for example, 10 plaques in the periventricular area, does not fulfill the criteria for dissemination in space, while two plaques located in two different locations (mentioned above) fulfill these criteria. Therefore, although the number of plaques is important in MS diagnosis, finding plaques in different anatomic sites is even more important (i.e., finding only one plaque in different anatomic sites is more important than finding multiple plaques in the same location).

When comparing different MRI sequences for the detection of CLs in a group of suspected MS patients, it is even more important to compare the frequency of patients with at least one cortical plaque rather than merely compare the mean number of plaques between two sequences. Therefore, we compared the sequences regarding the presence or absence of plaques, in addition to the mean number of plaques; the results are presented in Table 5. For both radiologists, the percentage of patients who showed at least one plaques in PSIR and did not show plaque in T2W-TSE was higher (at least equal to 26.3\%) than the patients who did no show plaque in PSIR and showed at least one plaques in T2W-TSE in both IC and LC regions. Therefore, compared to T2W-TSE, PSIR could potentially increase the MS diagnosis in more than $25 \%$ of the patients; however, such a conclusion cannot be made solely based on the comparison of the mean plaque counts.

Similarly, when we compared the presence of plaques (at least one) in PSIR and FLAIR in the IC and LC regions, we observed similar findings; in the PSIR sequence, at least $26.3 \%$ of the patients showed plaques, while they did not show any plaques in FLAIR; this finding could not be obtained based on the mean plaque comparison. It shows that PSIR, compared to T2W-TSE and FLAIR, can significantly improve the MS diagnosis in a group of suspected MS patients when evaluating the cortical regions.

The present study had some limitations. First, the sample size was small in this study. Second, most of our patients were diagnosed with RR-MS; it should be noted that in this subgroup of MS patients, the probability of CL is lower than that of primary progressive and secondary progressive MS subtypes. Third, we did not evaluate the relationship between the CL burden and the clinical course of the disease. We suggest using a 3D PSIR for the improved detection of CLs and also improved disease prediction, as lesions in the GM can lead to clinical disabilities.

In conclusion, a larger number of IC and LC lesions can be detected using PSIR compared to FLAIR and T2W-TSE sequences. Since the precise detection of IC lesions is important in monitoring cortical injuries and disease progression in MS patients over a long-term follow-up, we recommend adding PSIR sequence into the routine MR protocol for MS patients, especially those who are suspected of cog- nitive impairments.

\section{Footnotes}

Authors' Contribution: Hassan Hashemi, assessment, interpretation, and acquisition of imaging data, critical revision for important intellectual content, and final approval of the version to be published; Maryam Mohammadzadeh, assessment, interpretation, and acquisition of imaging data, critical revision for important intellectual content, and final approval of the version to be published; Mohammad Hossein Dianat, assessment, critical revision for important intellectual content, and final approval of the version to be published; Amir Reza Azimi, acquisition of data, critical revision for important intellectual content, and final approval of the version to be published; Hamed Naghibi, study conception, study design, acquisition of data, drafting the manuscript, critical revision for important intellectual content, and the final approval of the version to be published; Madjid Shakiba, study design, statistical data analysis, drafting the manuscript, critical revision for important intellectual content, and final approval of the version to be published; and Kavous Firouznia, study conception, study design, assessment, interpretation, and acquisition of imaging data, critical revision for important intellectual content, and final approval of the version to be published.

Conflict of Interests: The authors declare no relationships with any companies whose products or services may be related to the subject matter of this article.

Ethical Approval: Code: IR.TUMS.VCR.REC.1397.238.

Funding/Support: No funding was received for this study. Informed Consent: All subjects provided a written informed consent form before the study.

\section{References}

1. Harel A, Ceccarelli A, Farrell C, Fabian M, Howard J, Riley C, et al. Phasesensitive inversion-recovery MRI improves longitudinal cortical lesion detection in progressive MS. PLoS One. 2016;11(3). e0152180. doi: 10.1371/journal.pone.0152180. [PubMed: 27002529]. [PubMed Central: PMC4803340].

2. Sethi V, Yousry TA, Muhlert N, Ron M, Golay X, Wheeler-Kingshott C, et al. Improved detection of cortical MS lesions with phase-sensitive inversion recovery MRI. J Neurol Neurosurg Psychiatry. 2012;83(9):87782. doi: 10.1136/jnnp-2012-303023. [PubMed: 22807559].

3. Nelson F, Poonawalla A, Datta S, Wolinsky J, Narayana P. Is 3D MPRAGE better than the combination DIR/PSIR for cortical lesion detection at 3T MRI? Mult Scler Relat Disord. 2014;3(2):253-7. doi: 10.1016/j.msard.2013.10.002. [PubMed: 25878013].

4. Nelson F, Poonawalla AH, Hou P, Huang F, Wolinsky JS, Narayana PA. Improved identification of intracortical lesions in multiple sclerosis with phase-sensitive inversion recovery in combination with fast double inversion recovery MR imaging. AJNR Am J Neuroradiol. 2007;28(9):1645-9. doi: 10.3174/ajnr.A0645. [PubMed: 17885241]. [PubMed Central: PMC8134176]. 
5. Bisecco A, Rocca MA, Pagani E, Mancini L, Enzinger C, Gallo A, et al. Connectivity-based parcellation of the thalamus in multiple sclerosis and its implications for cognitive impairment: A multicenter study. Hum Brain Mapp. 2015;36(7):2809-25. doi: 10.1002/hbm.22809. [PubMed: 25873194]. [PubMed Central: PMC6869750].

6. Preziosa P, Rocca MA, Pagani E, Stromillo ML, Enzinger C, Gallo A, et al. Structural MRI correlates of cognitive impairment in patients with multiple sclerosis: A Multicenter Study. Hum Brain Mapp. 2016;37(4):1627-44. doi: 10.1002/hbm.23125. [PubMed: 26833969]. [PubMed Central: PMC6867484].

7. Damjanovic D, Valsasina P, Rocca MA, Stromillo ML, Gallo A, Enzinger $\mathrm{C}$, et al. Hippocampal and deep gray matter nuclei atrophy is relevant for explaining cognitive impairment in MS: A multicenter study. AJNR Am J Neuroradiol. 2017;38(1):18-24. doi: 10.3174/ajnr.A4952. [PubMed: 27686487]. [PubMed Central: PMC7963669].

8. Geurts JJ, Pouwels PJ, Uitdehaag BM, Polman CH, Barkhof F, Castelijns JA. Intracortical lesions in multiple sclerosis: Improved detection with 3D double inversion-recovery MR imaging. Radiology. 2005;236(1):254-60. doi: 10.1148/radiol.2361040450. [PubMed: 15987979].

9. Geurts JJ, Roosendaal SD, Calabrese M, Ciccarelli O, Agosta F, Chard DT, et al. Consensus recommendations for MS cortical lesion scoring using double inversion recovery MRI. Neurology. 2011;76(5):418-24. doi: 10.1212/WNL.ob013e31820a0cc4. [PubMed: 21209373].

10. Calabrese M, De Stefano N, Atzori M, Bernardi V, Mattisi I, Barachino $\mathrm{L}$, et al. Detection of cortical inflammatory lesions by double inversion recovery magnetic resonance imaging in patients with multiple sclerosis. Arch Neurol. 2007;64(10):1416-22. doi: 10.1001/archneur.64.10.1416. [PubMed: 17923625].

11. Naghibi H, Shekarchi B, Bagheri H, Azimi AR, Shakiba M. Comparison of phase sensitive inversion recovery MRI with T2W-TSE and STIR in the detection of cervical multiple sclerosis lesions. Iran J Radiol. 2019;16(2). e84059. doi: 10.5812/iranjradiol.84059.

12. Thompson AJ, Banwell BL, Barkhof F, Carroll WM, Coetzee T, Comi $\mathrm{G}$, et al. Diagnosis of multiple sclerosis: 2017 revisions of the Mc-
Donald criteria. Lancet Neurol. 2018;17(2):162-73. doi: 10.1016/S14744422(17)30470-2. [PubMed: 29275977].

13. Tallantyre EC, Morgan PS, Dixon JE, Al-Radaideh A, Brookes MJ, Morris PG, et al. 3 Tesla and 7 Tesla MRI of multiple sclerosis cortical lesions. J Magn Reson Imaging. 2010;32(4):971-7. doi: 10.1002/jmri.22115. [PubMed: 20882628].

14. Nelson F, Datta S, Garcia N, Rozario NL, Perez F, Cutter G, et al. Intracortical lesions by $3 \mathrm{~T}$ magnetic resonance imaging and correlation with cognitive impairment in multiple sclerosis. Mult Scler. 2011;17(9):11229. doi: 10.1177/1352458511405561. [PubMed: 21543552]. [PubMed Central: PMC3151473].

15. Elnekeidy AM, Kamal MA, Elfatatry AM, Elskeikh ML. Added value of double inversion recovery magnetic resonance sequence in detection of cortical and white matter brain lesions in multiple sclerosis. Egypt J Radiol Nucl Med. 2014;45(4):1193-9. doi: 10.1016/j.ejrnm.2014.06.010.

16. Moran PR, Kumar NG, Karstaedt N, Jackels SC. Tissue contrast enhancement: Image reconstruction algorithm and selection of TI in inversion recovery MRI. Magn Reson Imaging. 1986;4(3):229-35. doi: 10.1016/0730-725x(86)91062-3. [PubMed: 3669934].

17. Favaretto A, Poggiali D, Lazzarotto A, Rolma G, Causin F, Gallo P. The parallel analysis of phase sensitive inversion recovery (PSIR) and double inversion recovery (DIR) images significantly improves the detection of cortical lesions in multiple sclerosis (MS) since clinical onset. PLoS One. 2015;10(5). e0127805. doi: 10.1371/journal.pone.0127805. [PubMed: 26010425]. [PubMed Central: PMC4444021].

18. Wattjes MP, Lutterbey GG, Gieseke J, Traber F, Klotz L, Schmidt S, et al. Double inversion recovery brain imaging at 3T: Diagnostic value in the detection of multiple sclerosis lesions. AJNR Am J Neuroradiol. 2007;28(1):54-9. [PubMed: 17213424]. [PubMed Central: PMC8134107].

19. Sethi V, Muhlert N, Ron M, Golay X, Wheeler-Kingshott CA, Miller DH, et al. MS cortical lesions on DIR: Not quite what they seem? PLoS One. 2013;8(11). e78879. doi: 10.1371/journal.pone.0078879. [PubMed: 24244381]. [PubMed Central: PMC3823986]. 